\title{
Postoperative radiation in esophageal squamous cell carcinoma and target volume delineation
}

\author{
This article was published in the following Dove Press journal: \\ OncoTargets and Therapy \\ II July 2016 \\ Number of times this article has been viewed
}

\section{Yingming Zhu* \\ Minghuan $\mathrm{Li}^{*}$ \\ Li Kong \\ Jinming Yu}

Department of Radiation Oncology, Shandong Cancer Hospital and Institute, Shandong University, Jinan, Shandong, People's Republic of China

*These authors contributed equally to this work
Correspondence: Jinming Yu Department of Radiation Oncology, Shandong Cancer Hospital and Institute, Shandong University, 440 Jiyan Road, Jinan 250II7, Shandong, People's Republic of China

Tel +8653 I 87984729

Fax +86 53I 87984079

Email chinasdyjm@sohu.com

\begin{abstract}
Esophageal cancer is the sixth leading cause of cancer death worldwide, and patients who are treated with surgery alone, without neoadjuvant therapies, experience frequent relapses. Whether postoperative therapies could reduce the recurrence or improve overall survival is still controversial for these patients. The purpose of our review is to figure out the value of postoperative adjuvant therapy and address the disputes about target volume delineation according to published data. Based on the evidence of increased morbidity and disadvantages on patient survival caused by postoperative chemotherapy or radiotherapy (RT) alone provided by studies in the early 1990s, the use of postoperative adjuvant therapies in cases of esophageal squamous cell carcinoma has diminished substantially and has been replaced gradually by neoadjuvant chemoradiation. With advances in surgery and RT, accumulating evidence has recently rekindled interest in the delivery of postoperative RT or chemoradiotherapy in patients with stage T3/T4 or N1 (lymph node positive) carcinomas after radical surgery. However, due to complications with the standard radiation field, a nonconforming modified field has been adopted in most studies. Therefore, we analyze different field applications and provide suggestions on the optimization of the radiation field based on the major sites of relapse and the surgical non-clearance area. For upper and middle thoracic esophageal carcinomas, the bilateral supraclavicular and superior mediastinal areas remain common sites of recurrence and should be encompassed within the clinical target volume. In contrast, a consensus has yet to be reached regarding lower thoracic esophageal carcinomas; the "standard" clinical target volume is still recommended. Further studies of larger sample sizes should focus on different recurrence patterns, categorized by tumor locations, refined classifications, and differing molecular biology, to provide more information on the delineation of target volumes.
\end{abstract}

Keywords: adjuvant therapy, delineation of clinical target volume, postoperative locoregional recurrence, postoperative radiation, thoracic esophageal squamous cell carcinoma

\section{Introduction}

Esophageal cancer (EC) is the sixth leading cause of cancer-related death worldwide and is characterized by its extreme aggression and poor prognosis. ${ }^{1,2}$ Globally, squamous cell carcinoma (SCC) is the most common type of EC especially in the "Asian EC belt" that extends from northeast China to the Middle East. ${ }^{3}$ Esophagectomy remains the cornerstone of treatment for early-stage and locally advanced thoracic esophageal SCC (ESCC). ${ }^{4}$ Resection rates range from 19\% to 64\%, while 5-year survival ranges from $10 \%$ to $55 \%$ depending on the stage. ${ }^{5-7}$ Although advances in surgical and anesthetic techniques and improvements in perioperative management continue to reduce the postoperative mortality rate, this reduction has failed to translate into a significant benefit with regard to long-term survival. This is due to both high recurrence rates (34\%-79\%) and a short remission period (median, 14 months). ${ }^{8,9}$ Attempts have been 
made to correct this through an increase in the radical nature of the nodal dissection or through a combination of surgery with other treatment modalities, such as preoperative or postoperative chemoradiation.

The current guidelines of the National Comprehensive Cancer Network (NCCN) recommend neoadjuvant chemoradiation or definitive chemoradiation for all patients if the disease stage exceeds T1N0. ${ }^{10}$ However, the key evidence for neoadjuvant therapy comes primarily from trials in Western countries. ${ }^{11}$ Due to the growing distinction between the East and West in terms of the pathological types of EC, a large proportion of patients with adenocarcinoma included in the trials are not in accord with the situation in Asian countries. ${ }^{12}$ In addition, several studies have reported that neoadjuvant therapies for ESCC may increase the risk of postoperative morbidity or perioperative mortality, in addition to fear of disease progression and missing the opportunity for surgical therapy; thus, a considerable number of patients with locally advanced thoracic ESCC in the People's Republic of China tended not to conform with the NCCN guidelines and undergo surgery as an initial treatment. ${ }^{13-15}$ For these patients, even complete resection of the tumor is often inadequate. The search for a suitable adjuvant therapy after esophagectomy is particularly important for these patients with a high risk of recurrence.

\section{Trends in the use of postoperative radiotherapy after resection of ESCC}

Radiotherapy (RT) and chemotherapy represent the main treatment modalities in ESCC apart from surgery. ${ }^{16}$ Several molecular targeted agents, such as cetuximab, which have shown advantages in cancer therapy, are still in clinical trials, mainly for advanced or recurrence ESCC. ${ }^{17}$ Compared with neoadjuvant therapies, there have been relatively few studies of adjuvant therapies. In part, this may be because the typically debilitating condition of patients who undergo esophagectomies makes them unable to withstand the toxicity of postoperative treatments. ${ }^{15}$ Furthermore, the unsuccessful studies of postoperative RT (PORT) or postoperative chemotherapy (POCT) alone in the 20th century shifted the focus from adjuvant to neoadjuvant therapy. ${ }^{18-20}$ However, the lack of benefit of PORT alone was felt to be, in large part, a result of inappropriate patient selection and outdated techniques and equipment. For example, two of the studies ${ }^{18,20}$ included patients with positive celiac nodes (stage M1a) who were supposedly at a much higher risk for distant failure and were, thus, less likely to benefit from adjuvant RT alone; in these studies, irradiation was based on two-dimensional (2D) treatment plan using simple anteroposterior-posteroanterior techniques. The rate of local recurrence was lower in three of the PORT versus surgeryalone trials, but two trials noted that increased morbidity such as radiation fibrosis of the lung, noncancerous pericardial and pleural effusion, and alimentary tract hemorrhage counteracted any benefits. ${ }^{18,19}$ Moreover, one of the trials adopted hypofractionation schedules (49.5 Gy at $3.5 \mathrm{~Gy} /$ fraction), which may have caused severe complications and increased mortality. ${ }^{19}$ The actual dose distribution to organs at risk could not be evaluated, due to the technical limitations of 2DRT. More importantly, two of the studies included large proportions of patients who underwent palliative surgery, which affected the results greatly. ${ }^{19,20}$ Obviously, these studies conducted from the late 1980s to the early 1990s are not representative of the patients who receive therapy today.

Continued advancements in minimally invasive esophagectomies, chemotherapeutics, RT technology, and nutritional support over the past few decades have rendered patients a better ability to tolerate adjuvant therapy. Recent research has frequently showed the potential efficacy of POCT, PORT, and postoperative chemoradiotherapy (POCRT). ${ }^{21-24}$ The advantages of postoperative treatments over neoadjuvant therapies are based on accurate pathological staging; thus, the risk of overtreatment is minimized. Recent studies have suggested that POCT alone significantly improves disease-free survival (DFS), especially for nodepositive ESCC, but showed no significant benefit in overall survival (OS) in most studies. ${ }^{21,24}$ In contrast, accumulating studies of PORT or POCRT demonstrated OS and DFS benefits compared with surgery alone in selected locally advanced patients. ${ }^{14,15,22,23,25,26}$ Xiao et al $^{27}$ reported no survival benefit for the entire cohort with the addition of PORT. When stratifying based on stage, however, there was a significant survival benefit with PORT for stage III patients. Other studies on PORT also draw similar conclusions. ${ }^{28,29}$ Wang et $\mathrm{al}^{22}$ focused on ESCC with extracapsular lymph node extension, which is associated with a poor prognosis; they found that postoperative adjuvant chemoradiotherapy significantly reduced local recurrence rates and improved the DFS and OS compared with surgery alone. One study even suggested that the survival benefits provided by POCRT may catch up with neoadjuvant chemoradiotherapy. ${ }^{15}$ Even for T2N0M0 patients, a study showed that adjuvant RT could significantly improve survival for the patients with a high risk of poor prognosis. ${ }^{14}$ However, PORT for patients with T2N0M0 EC is still controversial and should be adopted with caution. ${ }^{30}$ 
In summary, the addition of PORT can significantly improve the prognosis of patients with locally advanced ESCC (stage T3/T4 or lymph node-positive carcinoma).

Improvement of PORT in ESCC may be due to the advances in nutritional support and esophagectomies rather than RT technology. The study by Xiao et $\mathrm{al}^{23}$ used 2D treatment planning, while other studies either did not provide detailed information about radiation techniques or contained both 2DRT and three-dimensional (3D) RT techniques but did not compare outcomes or toxicity. ${ }^{26,28,29}$ In view of the popularity of computed-tomography-based simulation in RT for stage III non-small-cell lung cancer (NSCLC) in the US (13\% in 1998 to $77.6 \%$ in 2005) $)^{31}$ and the years of inclusion, at least some of the patients were assessed with 2D treatment planning. Despite relatively few studies comparing 2DRT and 3DRT in PORT for EC, undeniably, 3D conformal RT (3DCRT), intensity-modulated RT (IMRT), and image-guided RT can provide better dosimetry than conventional 2D radiation, with improved target coverage and sparing of surrounding normal tissues. 3DRT has been independently associated with a survival advantage and a higher local tumor control rate in nasopharyngeal carcinoma and NSCLC. ${ }^{32,33}$ Moreover, studies at the MD Anderson Cancer Center (Houston, TX, USA) showed that dosimetric advantages of IMRT may translate into clinical benefits by reducing the incidence of cardiac deaths in definitive chemoradiotherapy of ESCC. ${ }^{34}$ In addition, a recent study evaluated the intrathoracic stomach toxicity of PORT in patients with ESCC after esophagectomy, followed by gastric conduit reconstruction, and revealed that only $15(14.3 \%)$ patients suffered from grade 2 and higher late toxicities. ${ }^{35}$ Although studies comparing different techniques in PORT are rare, these results provide the impetus for further study of advanced RT technologies in PORT for EC. By comparison, significant technological improvements and optimization of the doses and fractionation regimens have dramatically increased the OS of patients who receive PORT and have changed the treatment modality of choice in NSCLC. ${ }^{36,37}$ Similarly, postmastectomy RT in breast cancer was long considered deleterious until the publication of two large randomized studies ${ }^{38,39}$ that featured the use of more modern RT techniques, showing clear improvements in both DFS and OS. ${ }^{40}$ Thus, innovations in technology may reform treatment modalities. Proton beam therapy offers advantages over IMRT in terms of normal tissue sparing and may result in clinically significant reductions in acute and long-term treatment-related toxicities. However, reported clinical experiences for proton beam therapy have been limited to locally recurrent or persistent EC, and there is no report of proton beam therapy in postoperative prophylactic radiation to date. ${ }^{41}$ Helical tomotherapy is an important technological advance in terms of modularity and safety for complex RT techniques. ${ }^{42}$ However, the techniques are currently of limited availability in postoperative prophylactic RT in the People's Republic of China, due to the high investment costs in equipment and personnel. PORT versus surgery alone studies are summarized in Table 1.

\section{Current situation regarding PORT clinical target volume delineation}

Currently, no consensus has been reached with regard to the optimal extent of PORT clinical target volume (CTV) to treat radically resected ESCC. Based on the longitudinally drained submucosal lymphatic net and the probability of skipping lymph node metastasis in thoracic EC, standard PORT CTV should encompass the tumor bed, the bilateral supraclavicular areas, the mediastinal lymph nodes, and the cardia and left gastric lymph nodes..$^{23,43-45}$ Such extensive fields can effectively lower the chance of local recurrence within the range of radiation portals. ${ }^{23}$ Although several studies have demonstrated the safety and feasibility of extended-volume RT, ${ }^{46}$ such an extensive irradiation field frequently causes severe gastrointestinal and systemic reactions, and many patients who have poor tolerance are required to suspend the treatment or reduce the radiation dose. Some patients even experience severe radiation-induced fibrosis of the lung, noncancerous pericardial and pleural effusions, and alimentary tract hemorrhages. ${ }^{23}$ To date, few studies have strictly adopted the standard radiation field without optimization. In the prospective randomized study by Xiao et al, ${ }^{23}$ a standard CTV was adopted, and $\sim 40 \%$ of the patients developed reactions, such as nausea, anorexia, and leukopenia to varying degrees soon after treatment; $6 \%$ developed chronic toxic effects, such as radiation-induced lung injury (ie, fibrosis) and noncancerous pericardial and pleural effusions. Moreover, two patients experienced gastrointestinal bleeding. Such serious complications may be responsible for the absence of a survival advantage in certain patients, and two main factors were responsible, which include outdated anteroposterior-posteroanterior techniques and an overextensive irradiation field. In theory, advanced technologies such as 3DCRT and IMRT can reduce the RT-related toxicity by better dose delivery to the precisely defined planning target volume, as mentioned earlier. However, recent reports of PORT with 3DCRT or IMRT techniques in ESCC rarely adopted a standard radiation field. ${ }^{14,47,48}$ This is probably 


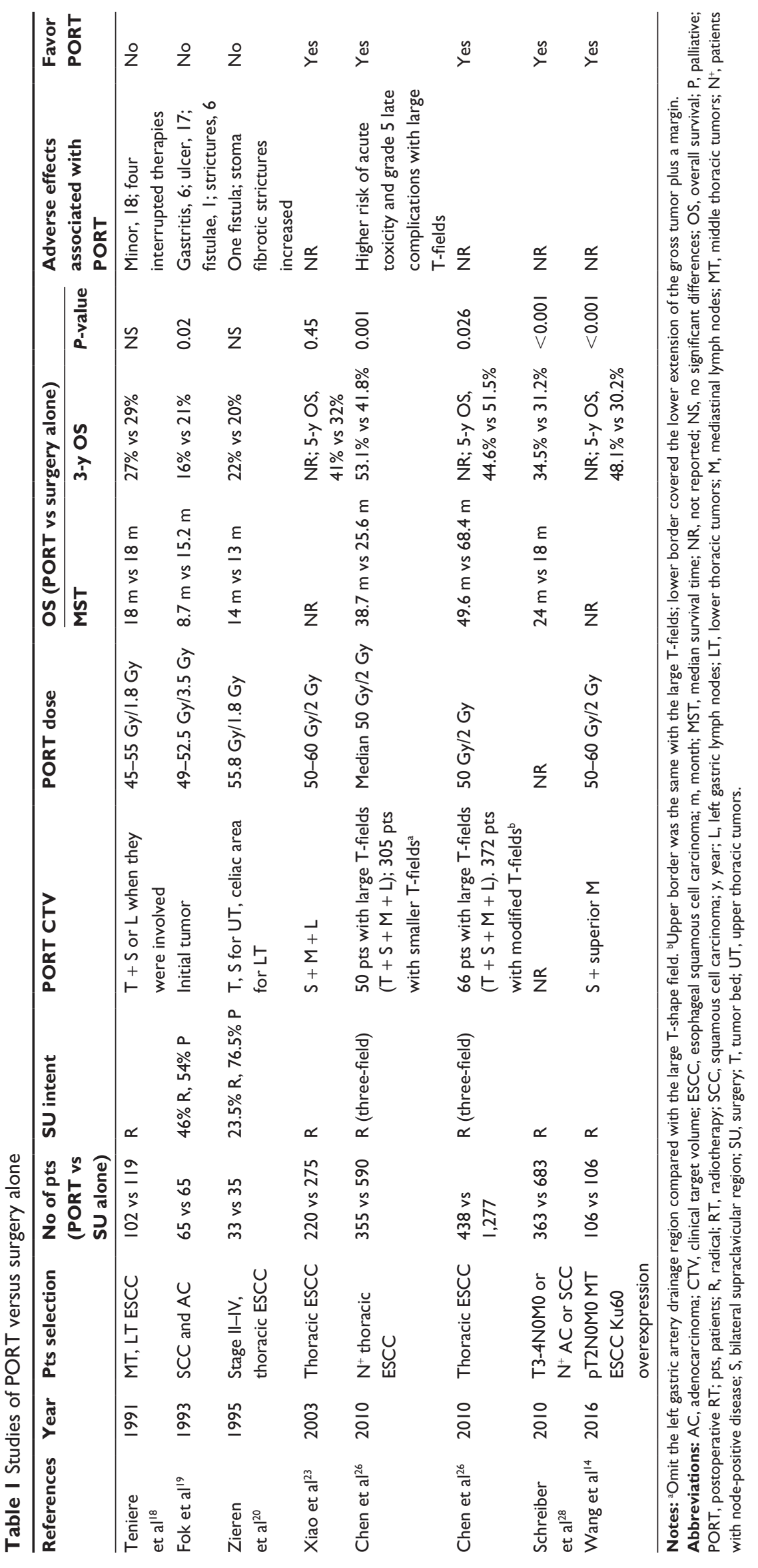


because the clinicians are reluctant to attempt to change the radiation fields used in times of 2DRT even though advanced technologies reduce the RT-related toxicity. ${ }^{49}$ Thus, there is a lack of data on the evaluation of toxicity with a standard radiation field based on the dose-volume analysis of normal organs using dose-volume histogram parameters. Reevaluation of the efficacy and side effects of PORT with a standard radiation field using new technology is needed.

The exact rate of lymph node skipping metastasis is difficult to evaluate. Some studies found the probability of skipping metastasis in EC to be only $5 \%-10 \% .^{50}$ This may provide an opportunity to optimize the irradiation field and to reduce the adverse effects. Attempts have been made since the 20th century to optimize the irradiation field. Different ranges of PORT CTV were used in three Phase III randomized controlled trials (mediastinal, supraclavicular, and celiac area with celiac lymph node invasion; initial tumor bed only; and the entire mediastinum and the region of local lymphatic drainage, respectively, in the three trials). ${ }^{18-20}$ Severe radiation-related complications were observed during the follow-up period in two trials including fatal gastrointestinal bleeding and anastomotic stricture. However, inadequate patient inclusion and the obsolete techniques in these studies, as mentioned earlier, make these results less valuable. So far, relatively little work has concentrated on the development of guidelines with regard to which sites should be included or excluded. The range of the radiation field differs across studies of PORT without chemotherapy which have included 1) the bilateral supraclavicular areas and the entire mediastinum; ${ }^{18}$ 2) the bilateral supraclavicular areas, the entire mediastinum, and the left gastric lymph nodes ${ }^{51}$ 3) the tumor bed alone $;{ }^{19}$ and 4 ) a T-shaped field that included the bilateral lower cervical and supraclavicular areas, as well as the upper portion of the mediastinum ${ }^{27}$ thus, the dispute centers on the extent of lymph node irradiation can be seen. Two retrospective studies focused on a comparison of the standard CTV with regional fields. ${ }^{50,51}$ Qiao et al ${ }^{51}$ compared an extended RT that covered the supraclavicular nodes, anastomotic sites, and tumor bed to regional fields that were confined to the tumor bed and the lymph nodes in the immediate region of the primary lesion. Although they did not analyze the DFS and recurrence patterns as limited imaging studies were conducted during the follow-up, the OS rates were not compromised in the regional portal group. Thus, regional portal fields should be considered due to the identical effects achieved in a larger field, while an extended volume may not be necessary. Lu et a ${ }^{50}$ reviewed 204 patients with thoracic ESCC who had received PORT after radical surgery.
The patients were classified into four groups according to the radiation field: the entire mediastinum alone, mediastinum + the bilateral supraclavicular area, mediastinum + the left gastric area, and mediastinum + bilateral supraclavicular area + left gastric area (standard CTV). None of the patients with disease in the upper third of the esophagus and only one of 83 middle cases without radiation of the left gastric area were shown to have abdominal lymph node metastasis. Similarly, the rate of metastasis of the supraclavicular lymph node in patients with disease in the lower third of the esophagus was $2 / 61$, whether the bilateral supraclavicular area was irradiated or not. They concluded that it may be unnecessary to irradiate the left gastric area when the primary lesion is located in the upper and middle portion of the esophagus. Likewise, the bilateral supraclavicular area may be unnecessarily irradiated in cases when the disease is present in the lower and middle lower thirds. Moreover, tumor $\mathrm{T}$ and $\mathrm{N}$ stages are independent prognostic factors for DFS rather than the extent of irradiation. In Chen et al's study, ${ }^{29}$ the standard CTV was modified to a smaller T-field that excluded the left gastric artery drainage region and only covered the bilateral supraclavicular areas, mediastinum, and tumor bed due to unacceptably high complication rates. Significant reductions in acute and late RT complications, such as grade 5 gastric bleeding, as well as cardiac and pulmonary complications, were observed in patients who received smaller T-field irradiation. Moreover, the omission of that area did not result in more failure in the celiac region or compromise with regard to the 5-year survival rate. However, despite these studies that showing no obviously compromised effect or additional recurrent risk, there has always been some concern about recurrence in regional lymph nodes outside of the irradiation field when the field is reduced.

As the studies mentioned earlier indicate, in addition to the dosage and fraction, portal designation plays a pivotal role in the OS and the occurrence of complications. An issue of growing concern is how the portal designation can be optimized without a concomitant compromise in the outcome. The optimal PORT CTV should be limited to high-risk areas of postoperative recurrence, so that the occurrence of severe adverse effects is reduced. Portal designation should be considered from the following perspectives: 1) extent of surgical lymph node clearance, 2) site of relapse after radical esophagectomy without preoperative chemotherapy and radiation, and 3) stage and location of the primary lesion. As Table 1 shows, the primary dispute in the range of CTV concerns the range of the prophylactic lymphoid drainage area. 


\section{Optimization of PORT CTV}

Among the three perspectives mentioned earlier, the extent of surgical lymph node clearance and the site of relapse are interdependent. The complex anatomy and the abundance of nerves and large blood vessels, especially the tight connection between the recurrent laryngeal nerve and surrounding lymph nodes in the upper mediastinum areas, greatly increase the technical difficulty of surgery and may result in the omission of lymph nodes during lymphadenectomy ${ }^{52}$ Correspondingly, several studies showed that the bilateral supraclavicular and superior mediastinal areas had the highest recurrence rates, regardless of the primary tumor site. ${ }^{52,53}$ Japanese medical professionals prefer to extend the lymphadenectomy to the cervical region in cases of upper/middle thoracic EC since a nationwide survey in 1991 showed a dramatic difference in 5-year survival rates between a three-field lymphadenectomy (3-FL) and a two-field lymphadenectomy (2-FL). ${ }^{54}$ However, recent studies showed that 3-FL only reduced the local recurrence rate of the superior mediastinum nodes slightly compared with modern 2-FL and that the bilateral supraclavicular and mediastinal nodes remained as the major locoregional recurrence sites. ${ }^{55,56}$ From this point of view, 3-FL or 2-FL has no obvious impact on the PORT CTV. As mentioned earlier, the dispute in PORT CTV delineation is focused on the extent of lymph node irradiation. ${ }^{50,51}$ The pattern of regional lymph node metastasis after surgery alone is the most important factor in the guidance for the establishment of the PORT CTV. The pattern may be influenced by primary tumor location, tumor, node, and metastasis (TNM) stage, with or without POCT and other factors. We address these points in detail in the following sections.

\section{Optimization of PORT CTV according to different location of primary lesion}

Various studies have found that the incidence of metastasis in the regional lymph nodes was associated with the primary tumor site. ${ }^{57,58}$ For example, upper thoracic esophageal tumors frequently metastasize to the cervical nodes, while lower thoracic tumors are more prone to metastasize to nodes in the upper abdominal cavity. Thus, a special relationship may exist between the tumor location and the mode of lymphatic recurrence.

\section{Upper and middle thoracic ESCC}

Various studies have suggested that recurrences of upper and middle thoracic esophageal carcinomas after radical surgery tend to occur in the bilateral supraclavicular areas and in the superior mediastinum..$^{52,53,57,58}$ Liu et al ${ }^{53}$ studied 414 patients retrospectively after radical esophagectomy without prior therapy or PORT and found that the supraclavicular and superior mediastinal lymph nodes showed the highest initial rates of recurrence of all locations after surgical resection. In fact, $79.4 \%$ of all recurrence sites across $76.3 \%$ of the patients were located in the bilateral supraclavicular areas and in the upper portion of the mediastinum. Thus, they proposed that a CTV consisting of the bilateral supraclavicular and superior mediastinal areas (rather than all lymphatic drainage regions) would be adequate for the vast majority of patients. In the study by Doki et al, ${ }^{57}$ the vertical location of a thoracic EC has been suggested to strongly affect the site of tumor recurrence after curative surgery. For upper and middle esophageal tumors, they reached a conclusion similar to that of Liu et al: $:^{53}$ recurrence in the cervical and upper mediastinal nodes was most frequent, although $8 \%$ of the enrolled patients were diagnosed with non-squamous thoracic esophageal carcinomas. Two other studies demonstrated that apart from the supraclavicular and superior mediastinal areas, station 7 lymph nodes also had high metastasis rates. In Cai and Xin's study, ${ }^{58}$ the recurrence rate of station 7 lymph nodes was $37.2 \%$ for middle thoracic ESCC, while in Li et al's study ${ }^{52}$ it was $34.1 \%$. Both studies recommended that subcarinal regions should be encompassed within the target volume. In particular, a significant difference was identified in $\mathrm{Li}$ et al's study ${ }^{52}$ between right and left supraclavicular lymph node metastasis ( $31.7 \%$ vs $16.7 \%$, respectively, in the studies by $\mathrm{Cai}$ and $\mathrm{Xin}^{58}$ and $\mathrm{Li}$ et al, $\left.{ }^{52} P=0.005\right)$. The reason why the bilateral supraclavicular and superior mediastinal areas are at a high risk of recurrence is partly due to frequent metastasis of the upper and middle thoracic ESCC to the cervical nodes and difficulty in complete lymph node dissection due to the complex anatomy versus the lower mediastinum and upper abdominal sites that can be exposed more readily.

However, some other findings do not correspond exactly to the abovementioned results, and the precise area of highrisk recurrence remains controversial. ${ }^{9}$ Lee et $\mathrm{al}^{9}$ reported that $38 \%$ of patients with middle thoracic carcinomas still experienced recurrence in the abdominal nodes. However, according to the studies by Liu et al, ${ }^{53}$ Doki et al, ${ }^{57}$ and Cai and Xin,${ }^{58}$ the recurrence rates in abdominal nodes was $\sim 10 \%$ for middle thoracic tumors; this rate is actually lower in upper thoracic tumors $(0 \%-4 \%)$. The middle and lower mediastinum and upper abdominal areas can be exposed readily, and lymph node dissection in these regions is comparatively complete, compared with dissections of the lower neck and upper mediastinal regions. In Chen et al's study, ${ }^{26}$ no patient experienced failure in the abdomen, regardless of whether they received PORT and whether the coverage of the abdominal regions was minimized in a smaller T-field radiation that 
was delivered to most of the patients. Thus, they indicated that irradiation of the upper abdominal area was unnecessary. However, further prospective and randomized trials are still needed to investigate whether the upper abdominal lymph nodes should be included.

\section{Lower thoracic ESCC}

Similar to upper and middle thoracic ESCC, complete lymph node dissection of superior mediastinal lymph nodes is challenging due to the complicated anatomy in this area. Several studies showed that the bilateral supraclavicular and superior mediastinal areas had the highest recurrence rates in patients with lower thoracic ESCC. ${ }^{52,53}$ In contrast, $\mathrm{Cai}$ and $\mathrm{Xin}^{58}$ reported that the constituent ratio of upper abdominal nodes in lower thoracic ESCC recurrence was $36.2 \%$, significantly higher than for middle and upper thoracic EC. Few studies have addressed the recurrence pattern of lower thoracic ESCC after radical surgery alone. Whether locoregional recurrence patterns of lower esophageal tumors are centered on downward areas, such as the para-cardiac and left gastric nodes, or on upward areas, such as the bilateral supraclavicular and superior mediastinal areas remains understudied. Although SCC occurs equally often in the middle and lower esophagus, the percentage of lower esophageal tumors is lower than middle esophageal tumors in some studies; ${ }^{8,12,52,58}$ thus, the true recurrence pattern may be obscured by that of middle esophageal tumors. Currently, although the upward area seems more worthy to be irradiated due to more supporting evidence and difficulty in delineating unclearly defined upper abdominal nodes, sufficient reasons for omitting the upper abdominal lymph nodes or bilateral supraclavicular and superior mediastinal areas for lower thoracic ESCC are lacking. Doki et $\mathrm{al}^{57}$ reported that distant metastases, such as those in the liver and abdominal para-aortic nodes, were the predominant sites of recurrence for lower thoracic tumors, suggesting that POCT or POCRT rather than PORT alone should be emphasized for lower esophageal tumors. Further investigations are needed to determine the recurrence pattern of lower thoracic ESCC. Currently, due to deeply divided opinions about recurrence patterns of lower thoracic ESCC after surgery alone, a standard CTV is still recommended for patients with lower thoracic ESCC.

\section{Optimization of PORT CTV with POCRT} Several studies have demonstrated that POCRT is significantly more effective than PORT alone in locally advanced ESCC, especially for patients with vascular emboli and other poor prognostic factors. ${ }^{15}$ Most studies adopted concurrent chemotherapy and RT with or without adjuvant chemotherapy. ${ }^{22,59,60}$ Delineation of the radiation field should be considered carefully with postoperative chemoradiation because treatment-related complications may add up. Apart from one study, ${ }^{46}$ which included only 15 patients with a standard radiation field and reported no serious treatment-related complications, all of the other reported studies modified the radiation field to cover only the tumor bed, with or without regional lymphatic areas. With improvements in accurate preoperative evaluations of primary tumors and modifications in surgical techniques for an $\mathrm{R} 0$ resection, most tumors can be removed completely. Thus, the importance of regional lymphatic areas has been highlighted. Several recent studies with large patient numbers included all covered regional lymphatic areas. $^{22,25,49,59}$ Similar to the studies that adopted only PORT without chemotherapy, there were obvious differences in regional lymphatic areas across the studies. ${ }^{22,25,46,49,59}$ Overall, most studies included the bilateral supraclavicular area and the mediastinum for upper and middle thoracic ESCC. ${ }^{22,25,49}$ For lower thoracic ESCC, much disagreement exists. Some studies $^{25,49}$ focused on the bilateral supraclavicular area and mediastinum, while others ${ }^{22,59}$ concentrated on celiac areas. Studies about recurrence patterns after POCT alone may provide some hints. In JCOG9204, ${ }^{24}$ the frequency of local recurrence in cervical and mediastinal nodes was slightly lower in the POCT group but still much higher than recurrence in abdominal lymph nodes. However, research in this area is limited, and further studies are needed to determine the effects of POCT in recurrence patterns.

\section{Optimization of PORT CTV according to TNM stage and other factors}

As mentioned earlier, the delivery of PORT is beneficial for patients with stage T3/T4 or N1 (lymph node positive) carcinoma, according to the sixth edition American Joint Committee on Cancer Staging Manual, ${ }^{61}$ after radical surgery with modern techniques. Compared with the sixth edition American Joint Committee on Cancer guidelines, ${ }^{61}$ the seventh edition incorporated non-anatomical factors, such as tumor differentiation and a refined $\mathrm{N}$ stage according to the number of regional lymph nodes containing metastases. ${ }^{62}$ Many studies have reported that lymph node recurrence increases dramatically with deeper invasion, higher number of lymph nodes with positive metastases, poorer tumor differentiation, and longer tumor length after esophagectomy. ${ }^{8,63}$ However, reported studies have not identified whether extensive portal RT or systemic therapy is more effective or which should be the preferred treatment for these patients. ${ }^{53}$ Biomarkers such as Ku80, VEGF, EGFR, and p53 are 
associated with poor prognosis; however, their relationship with recurrence patterns has rarely been studied. ${ }^{14,64}$ More refined research for different TNM stages and other nonanatomical prognostic factors, as well as comparisons of various treatment modalities, is needed.

In summary, multiple factors, such as tumor location, POCT, and TNM stage, influence postoperative recurrence in ESCC. Although the results from various studies are inconsistent, they can still provide insight into optimization of the PORT CTV. For upper and middle ECs, various studies have reported that the bilateral supraclavicular and superior mediastinum have the highest rates of recurrence and should be included within the PORT CTV. However, the recurrence pattern for lower third ESCC is still obscure, and the current conflicting evidence does not rule out any area. Thus, we still recommend a standard CTV for patients with lower thoracic ESCC who undergo PORT alone. POCT may also influence recurrence patterns in some way. Because increasing numbers of studies have shown the effects of postoperative chemoradiation, it is imperative that the impact of POCT on recurrence patterns is studied. Moreover, TNM stage and some other prognostic non-anatomical factors can affect patterns of recurrence, thereby affecting CTV delineation. Additional studies of larger sample sizes and with refined classifications are needed to analyze the patterns of lymph node recurrence to provide more information on the delineation of target volume.

\section{Conclusion}

The management of resectable EC has undergone a major evolution over the past three decades. Based on several trials in Western countries, strategies for preoperative chemoradiation have been recommended by NCCN guidelines for patients with locally advanced ESCC. However, for patients who do not follow the guidelines strictly without receiving neoadjuvant therapy, surgery alone is not enough, particularly for these patients with locally advanced disease due to high risk of recurrence and short remission period. Recent accumulating evidence indicates that patients with T3-T4 tumors and those with node-positive disease should receive PORT or POCRT, after radical esophagectomy without neoadjuvant therapy. The dosimetric advantages of modern RT techniques, such as 3DCRT and IMRT, can significantly reduce the adverse reaction of PORT. Although the standard CTV encompasses all of the high-risk recurrence areas, treatment-related toxicity in clinical practice has discouraged professionals from adopting this treatment modality on a large scale. It is important to optimize the radiation field to reduce the complications without compromising the effect. Based on sites of relapse and the surgical nonclearance area, for both upper and middle thoracic ESCC, at least the bilateral supraclavicular areas and the superior mediastinum should be included within the PORT CTV. For lower thoracic ESCC, disagreements in recurrence patterns have made it more difficult for professionals to optimize the radiation field. Standard CTV is still recommended currently. Other factors such as POCT, TNM staging, and tumor molecular biology may influence the recurrence pattern, but evidence is lacking. More studies on recurrence patterns based on factors to guide the optimization of PORT CTV and randomized studies that compare different ranges are required.

\section{Acknowledgment}

This review was approved by the Institutional Review Board of Shandong Cancer Hospital and Institute, Jinan, People's Republic of China.

\section{Disclosure}

The authors report no conflicts of interest in this work.

\section{References}

1. Torre LA, Bray F, Siegel RL, Ferlay J, Lortet-Tieulent J, Jemal A. Global cancer statistics, 2012. CA Cancer J Clin. 2015;65(2):87-108.

2. Ferlay J, Shin HR, Bray F, Forman D, Mathers C, Parkin DM. Estimates of worldwide burden of cancer in 2008: GLOBOCAN 2008. Int J Cancer. 2010;127(12):2893-2917.

3. Wheeler JB, Reed CE. Epidemiology of esophageal cancer. Surg Clin North Am. 2012;92(5):1077-1087.

4. Almhanna K, Shridhar R, Meredith KL. Neoadjuvant or adjuvant therapy for resectable esophageal cancer: is there a standard of care? Cancer Control. 2013;20(2):89-96.

5. Mariette C, Balon JM, Piessen G, Fabre S, Van Seuningen I, Triboulet JP. Pattern of recurrence following complete resection of esophageal carcinoma and factors predictive of recurrent disease. Cancer. 2003;97(7): $1616-1623$.

6. Mariette C, Finzi L, Fabre S, Balon JM, Van Seuningen I, Triboulet JP. Factors predictive of complete resection of operable esophageal cancer: a prospective study. Ann Thorac Surg. 2003;75(6):1720-1726.

7. Pennathur A, Gibson MK, Jobe BA, Luketich JD. Oesophageal carcinoma. Lancet. 2013;381(9864):400-412.

8. Nakagawa S, Kanda T, Kosugi S, Ohashi M, Suzuki T, Hatakeyama K. Recurrence pattern of squamous cell carcinoma of the thoracic esophagus after extended radical esophagectomy with three-field lymphadenectomy. J Am Coll Surg. 2004;198(2):205-211.

9. Lee SJ, Lee KS, Yim YJ, Kim TS, Shim YM, Kim K. Recurrence of squamous cell carcinoma of the oesophagus after curative surgery: rates and patterns on imaging studies correlated with tumour location and pathological stage. Clin Radiol. 2005;60(5):547-554.

10. National Comprehensive Cancer Network [homepage on the Internet]. Esophageal cancer clinical practice guidelines in oncology. Available from: https://www.nccn.org/professionals/physician_gls/f_guidelines. asp. Accessed July 1, 2016.

11. van Hagen P, Hulshof MC, van Lanschot JJ, et al. Preoperative chemoradiotherapy for esophageal or junctional cancer. N Engl J Med. 2012; 366(22):2074-2084.

12. Napier KJ, Scheerer M, Misra S. Esophageal cancer: a review of epidemiology, pathogenesis, staging workup and treatment modalities. World J Gastrointest Oncol. 2014;6(5):112-120. 
13. Kumagai K, Rouvelas I, Tsai JA, et al. Meta-analysis of postoperative morbidity and perioperative mortality in patients receiving neoadjuvant chemotherapy or chemoradiotherapy for resectable oesophageal and gastro-oesophageal junctional cancers. Br J Surg. 2014;101(4): 321-338.

14. Wang S, Wang Z, Yang Z, et al. Postoperative radiotherapy improves survival in stage pT2N0M0 esophageal squamous cell carcinoma with high risk of poor prognosis. Ann Surg Oncol. 2016;23(1):265-272.

15. Chen HS, Wu SC, Hsu PK, Huang CS, Liu CC, Wu YC. The prognostic impact of preoperative and postoperative chemoradiation in clinical stage II and III esophageal squamous cell carcinomas: a population based study in Taiwan. Medicine (Baltimore). 2015;94(25):e1002.

16. Vosmik M, Petera J, Sirak I, etal. Technological advances in radiotherapy for esophageal cancer. World J Gastroenterol. 2010;16(44):5555-5564.

17. Meng X, Wang J, Sun X, et al. Cetuximab in combination with chemoradiotherapy in Chinese patients with non-resectable, locally advanced esophageal squamous cell carcinoma: a prospective, multicenter phase II trail. Radiother Oncol. 2013;109(2):275-280.

18. Teniere P, Hay JM, Fingerhut A, Fagniez PL. Postoperative radiation therapy does not increase survival after curative resection for squamous cell carcinoma of the middle and lower esophagus as shown by a multicenter controlled trial. French University Association for Surgical Research. Surg Gynecol Obstet. 1991;173(2):123-130.

19. Fok M, Sham JS, Choy D, Cheng SW, Wong J. Postoperative radiotherapy for carcinoma of the esophagus: a prospective, randomized controlled study. Surgery. 1993;113(2):138-147.

20. Zieren HU, Muller JM, Jacobi CA, Pichlmaier H, Muller RP, Staar S. Adjuvant postoperative radiation therapy after curative resection of squamous cell carcinoma of the thoracic esophagus: a prospective randomized study. World J Surg. 1995;19(3):444-449.

21. Qin RQ, Wen YS, Wang WP, Xi KX, Yu XY, Zhang LJ. The role of postoperative adjuvant chemotherapy for lymph node-positive esophageal squamous cell carcinoma: a propensity score matching analysis. Med Oncol. 2016;33(4):31.

22. Wang ZW, Luan ZP, Zhang W, et al. Postoperative chemoradiotherapy improves survival in esophageal squamous cell cancer with extracapsular lymph node extension. Neoplasma. 2014;61(6):732-738.

23. Xiao ZF, Yang ZY, Liang J, et al. Value of radiotherapy after radical surgery for esophageal carcinoma: a report of 495 patients. Ann Thorac Surg. 2003;75(2):331-336.

24. Ando N, Iizuka T, Ide H, et al. Surgery plus chemotherapy compared with surgery alone for localized squamous cell carcinoma of the thoracic esophagus: a Japan Clinical Oncology Group Study - JCOG9204. J Clin Oncol. 2003;21(24):4592-4596.

25. Chen J, Pan J, Liu J, et al. Postoperative radiation therapy with or without concurrent chemotherapy for node-positive thoracic esophageal squamous cell carcinoma. Int J Radiat Oncol Biol Phys. 2013;86(4):671-677.

26. Chen J, Zhu J, Pan J, et al. Postoperative radiotherapy improved survival of poor prognostic squamous cell carcinoma esophagus. Ann Thorac Surg. 2010;90(2):435-442.

27. Xiao ZF, Yang ZY, Miao YJ, et al. Influence of number of metastatic lymph nodes on survival of curative resected thoracic esophageal cancer patients and value of radiotherapy: report of 549 cases. Int J Radiat Oncol Biol Phys. 2005;62(1):82-90

28. Schreiber D, Rineer J, Vongtama D, et al. Impact of postoperative radiation after esophagectomy for esophageal cancer. J Thorac Oncol. 2010; 5(2):244-250

29. Chen J, Pan J, Zheng X, et al. Number and location of positive nodes, postoperative radiotherapy, and survival after esophagectomy with three-field lymph node dissection for thoracic esophageal squamous cell carcinoma. Int J Radiat Oncol Biol Phys. 2012;82(1):475-482.

30. Martin JT, Worni M, Zwischenberger JB, et al. The role of radiation therapy in resected T2 N0 esophageal cancer: a population-based analysis. Ann Thorac Surg. 2013;95(2):453-458.

31. Chen AB, Neville BA, Sher DJ, Chen K, Schrag D. Survival outcomes after radiation therapy for stage III non-small-cell lung cancer after adoption of computed tomography-based simulation. J Clin Oncol. 2011;29(17):2305-2311
32. Lai SZ, Li WF, Chen L, et al. How does intensity-modulated radiotherapy versus conventional two-dimensional radiotherapy influence the treatment results in nasopharyngeal carcinoma patients? Int J Radiat Oncol Biol Phys. 2011;80(3):661-668.

33. Sher DJ, Koshy M, Liptay MJ, Fidler MJ. Influence of conformal radiotherapy technique on survival after chemoradiotherapy for patients with stage III non-small cell lung cancer in the National Cancer Data Base. Cancer. 2014;120(13):2060-2068.

34. Lin SH, Wang L, Myles B, et al. Propensity score-based comparison of long-term outcomes with 3-dimensional conformal radiotherapy vs intensity-modulated radiotherapy for esophageal cancer. Int J Radiat Oncol Biol Phys. 2012;84(5):1078-1085.

35. Liu Q, Cai XW, Fu XL, Chen JC, Xiang JQ. Tolerance and dose-volume relationship of intrathoracic stomach irradiation after esophagectomy for patients with thoracic esophageal squamous cell carcinoma. Oncotarget. 2015;6(31):32220-32227.

36. Billiet C, Decaluwe H, Peeters S, et al. Modern post-operative radiotherapy for stage III non-small cell lung cancer may improve local control and survival: a meta-analysis. Radiother Oncol. 2014;110(1):3-8.

37. Postoperative radiotherapy in non-small-cell lung cancer: systematic review and meta-analysis of individual patient data from nine randomised controlled trials. PORT Meta-analysis Trialists Group. Lancet. 1998;352(9124):257-263.

38. Overgaard M, Hansen PS, Overgaard J, et al. Postoperative radiotherapy in high-risk premenopausal women with breast cancer who receive adjuvant chemotherapy. Danish Breast Cancer Cooperative Group 82b Trial. N Engl J Med. 1997;337(14):949-955.

39. Ragaz J, Jackson SM, Le N, et al. Adjuvant radiotherapy and chemotherapy in node-positive premenopausal women with breast cancer. N Engl J Med. 1997;337(14):956-962.

40. Le Pechoux C, Dunant A, Pignon JP, et al. Need for a new trial to evaluate adjuvant postoperative radiotherapy in non-small-cell lung cancer patients with N2 mediastinal involvement. J Clin Oncol. 2007;25(7):e10-e11.

41. Chuong MD, Hallemeier CL, Jabbour SK, et al. Improving outcomes for esophageal cancer using proton beam therapy. Int J Radiat Oncol Biol Phys. 2016;95(1):488-497.

42. Fenwick JD, Tome WA, Soisson ET, Mehta MP, Rock Mackie T. Tomotherapy and other innovative IMRT delivery systems. Semin Radiat Oncol. 2006;16(4):199-208.

43. Bollschweiler E, Schroder W, Holscher AH, Siewert JR. Preoperative risk analysis in patients with adenocarcinoma or squamous cell carcinoma of the oesophagus. Br J Surg. 2000;87(8):1106-1110.

44. Prenzel KL, Bollschweiler E, Schroder W, et al. Prognostic relevance of skip metastases in esophageal cancer. Ann Thorac Surg. 2010;90(5): 1662-1667.

45. Tachibana M, Dhar DK, Kinugasa S, et al. Esophageal cancer with distant lymph node metastasis: prognostic significance of metastatic lymph node ratio. J Clin Gastroenterol. 2000;31(4):318-322.

46. Yu E, Tai P, Younus J, et al. Postoperative extended-volume externalbeam radiation therapy in high-risk esophageal cancer patients: a prospective experience. Curr Oncol. 2009;16(4):48-54.

47. Zhang W, Liu X, Xiao Z, et al. Efficacy of intensity-modulated radiotherapy for resected thoracic esophageal squamous cell carcinoma. Thorac Cancer. 2015;6(5):597-604.

48. Zhang W, Liu X, Xiao Z, et al. Postoperative intensity-modulated radiotherapy improved survival in lymph node-positive or stage III thoracic esophageal squamous cell carcinoma. Oncol Res Treat. 2015;38(3): 97-102.

49. Xu Y, Liu J, Du X, et al. Prognostic impact of postoperative radiation in patients undergoing radical esophagectomy for pathologic lymph node positive esophageal cancer. Radiat Oncol. 2013;8:116.

50. Lu JC, Tao H, Zhang YQ, et al. Extent of prophylactic postoperative radiotherapy after radical surgery of thoracic esophageal squamous cell carcinoma. Dis Esophagus. 2008;21(6):502-507.

51. Qiao XY, Wang W, Zhou ZG, Gao XS, Chang JY. Comparison of efficacy of regional and extensive clinical target volumes in postoperative radiotherapy for esophageal squamous cell carcinoma. Int J Radiat Oncol Biol Phys. 2008;70(2):396-402. 
52. Li CL, Zhang FL, Wang YD, et al. Characteristics of recurrence after radical esophagectomy with two-field lymph node dissection for thoracic esophageal cancer. Oncol Lett. 2013;5(1):355-359.

53. Liu Q, Cai XW, Wu B, Zhu ZF, Chen HQ, Fu XL. Patterns of failure after radical surgery among patients with thoracic esophageal squamous cell carcinoma: implications for the clinical target volume design of postoperative radiotherapy. PLoS One. 2014;9(5):e97225.

54. Isono K, Sato H, Nakayama K. Results of a nationwide study on the three-field lymph node dissection of esophageal cancer. Oncology. 1991; 48(5):411-420.

55. Shim YM, Kim HK, Kim K. Comparison of survival and recurrence pattern between two-field and three-field lymph node dissections for upper thoracic esophageal squamous cell carcinoma. J Thorac Oncol. 2010; 5(5):707-712.

56. Law S, Wong J. Two-field dissection is enough for esophageal cancer. Dis Esophagus. 2001;14(2):98-103.

57. Doki Y, Ishikawa O, Takachi K, et al. Association of the primary tumor location with the site of tumor recurrence after curative resection of thoracic esophageal carcinoma. World J Surg. 2005;29(6):700-707.

58. Cai WJ, Xin PL. Pattern of relapse in surgical treated patients with thoracic esophageal squamous cell carcinoma and its possible impact on target delineation for postoperative radiotherapy. Radiother Oncol. 2010;96(1): 104-107.
59. Hsu PK, Huang CS, Wang BY, Wu YC, Hsu WH. Survival benefits of postoperative chemoradiation for lymph node-positive esophageal squamous cell carcinoma. Ann Thorac Surg. 2014;97(5):1734-1741.

60. Rice TW, Adelstein DJ, Chidel MA, et al. Benefit of postoperative adjuvant chemoradiotherapy in locoregionally advanced esophageal carcinoma. J Thorac Cardiovasc Surg. 2003;126(5):1590-1596.

61. Greene FL. The American Joint Committee on Cancer: updating the strategies in cancer staging. Bull Am Coll Surg. 2002;87(7):13-15.

62. Rice TW, Blackstone EH, Rusch VW. 7th edition of the AJCC cancer staging manual: esophagus and esophagogastric junction. Ann Surg Oncol. 2010;17(7):1721-1724.

63. Mirinezhad SK, Somi MH, Seyednezhad F, et al. Survival in patients treated with definitive chemo-radiotherapy for non-metastatic esophageal cancer in north-west Iran. Asian Pac J Cancer Prev. 2013;14(3): 1677-1680.

64. Shang L, Liu HJ, Hao JJ, et al. A panel of overexpressed proteins for prognosis in esophageal squamous cell carcinoma. PLoS One. 2014; 9(10):e111045
OncoTargets and Therapy

\section{Publish your work in this journal}

OncoTargets and Therapy is an international, peer-reviewed, open access journal focusing on the pathological basis of all cancers, potential targets for therapy and treatment protocols employed to improve the management of cancer patients. The journal also focuses on the impact of management programs and new therapeutic agents and protocols on

\section{Dovepress}

patient perspectives such as quality of life, adherence and satisfaction. The manuscript management system is completely online and includes a very quick and fair peer-review system, which is all easy to use. Visit http://www.dovepress.com/testimonials.php to read real quotes from published authors. 